\title{
ON BASIC FUZZY KOROVKIN THEORY
}

\author{
GEORGE A. ANAstassiou
}

\begin{abstract}
We prove the basic fuzzy Korovkin theorem via a fuzzy ShishaMond inequality given here. This determines the degree of convergence with rates of a sequence of fuzzy positive linear operators to the fuzzy unit operator. The surprising fact is that only the real case Korovkin assumptions are enough for the validity of the fuzzy Korovkin theorem, along with a natural realization condition fulfilled by the sequence of fuzzy positive linear operators. The last condition is fulfilled by almost all operators defined via fuzzy summation or fuzzy integration.
\end{abstract}

\section{Introduction}

Motivation for this work are the references [1], [2], [5], [6]. Our results Theorems 3 and 4 are simple, basic and very general, directly transferring the real case of the convergence with rates of positive linear operators to the unit, to the fuzzy one. The same real assumptions are kept here in the fuzzy setting, and they are the only assumptions we make along with the very natural and general realization condition (1). Condition (1) is fulfilled by almost all example - fuzzy positive operators, that is, by most fuzzy summation and fuzzy integration operators. At each step of our work we provide an example to justify our method. To the best of our knowledge our theorems are the first general fuzzy Korovkin type results.

\section{Background}

We start with

Received by the editors: 09.06.2005

2000 Mathematics Subject Classification. 26E50, 41A17, 41A25, 41A36, 47S40.

Key words and phrases. Fuzzy positive linear operator, Fuzzy Korovkin theory, Fuzzy Shisha-Mond inequality, Fuzzy modulus of continuity. 
Definition 1 (see $[8]$ ). Let $\mu: \mathbb{R} \rightarrow[0,1]$ with the following properties:

(i) is normal, i.e., $\exists x_{0} \in \mathbb{R} ; \mu\left(x_{0}\right)=1$.

(ii) $\mu(\lambda x+(1-\lambda) y) \geq \min \{\mu(x), \mu(y)\}, \forall x, y \in \mathbb{R}, \forall \lambda \in[0,1]$ ( $\mu$ is called a convex fuzzy subset).

(iii) $\mu$ is upper semicontinuous on $\mathbb{R}$, i.e., $\forall x_{0} \in \mathbb{R}$ and $\forall \varepsilon>0, \exists$ neighborhood $V\left(x_{0}\right): \mu(x) \leq \mu\left(x_{0}\right)+\varepsilon, \forall x \in V\left(x_{0}\right)$.

(iv) The set $\overline{\operatorname{supp}(\mu)}$ is compact in $\mathbb{R}($ where $\operatorname{supp}(\mu):=\{x \in \mathbb{R} ; \mu(x)>0\})$.

We call $\mu$ a fuzzy real number. Denote the set of all $\mu$ with $\mathbb{R}_{\mathcal{F}}$.

E.g., $\mathcal{X}_{\left\{x_{0}\right\}} \in \mathbb{R}_{\mathcal{F}}$, for any $x_{0} \in \mathbb{R}$, where $\mathcal{X}_{\left\{x_{0}\right\}}$ is the characteristic function at $x_{0}$.

For $0<r \leq 1$ and $\mu \in \mathbb{R}_{\mathcal{F}}$ define $[\mu]^{r}:=\{x \in \mathbb{R}: \mu(x) \geq r\}$ and

$$
[\mu]^{0}:=\overline{\{x \in \mathbb{R}: \mu(x)>0\}} .
$$

Then it is well known [3] that for each $r \in[0,1],[\mu]^{r}$ is a closed and bounded interval of $\mathbb{R}$. For $u, v \in \mathbb{R}_{\mathcal{F}}$ and $\lambda \in \mathbb{R}$, we define uniquely the sum $u \oplus v$ and the product $\lambda \odot u$ by

$$
[u \oplus v]^{r}=[u]^{r}+[v]^{r}, \quad[\lambda \odot u]^{r}=\lambda[u]^{r}, \quad \forall r \in[0,1],
$$

where $[u]^{r}+[v]^{r}$ means the usual addition of two intervals (as subsets of $\mathbb{R}$ ) and $\lambda[u]^{r}$ means the usual product between a scalar and a subset of $\mathbb{R}$ (see, e.g., [4]). Notice $1 \odot u=u$ and it holds $u \oplus v=v \oplus u, \lambda \odot u=u \odot \lambda$. If $0 \leq r_{1} \leq r_{2} \leq 1$ then $[u]^{r_{2}} \subseteq[u]^{r_{1}}$. Actually $[u]^{r}=\left[u_{-}^{(r)}, u_{+}^{(r)}\right]$, where $u_{-}^{(r)} \leq u_{+}^{(r)}, u_{-}^{(r)}, u_{+}^{(r)} \in \mathbb{R}, \forall r \in[0,1]$. We denote $u \precsim v$ iff $u_{-}^{(r)} \leq v_{-}^{(r)}$ and $u_{+}^{(r)} \leq v_{+}^{(r)}$, all $r \in[0,1]$. Define

$$
D: \mathbb{R}_{\mathcal{F}} \times \mathbb{R}_{\mathcal{F}} \rightarrow \mathbb{R}_{+}
$$

by

$$
D(u, v):=\sup _{r \in[0,1]} \max \left\{\left|u_{-}^{(r)}-v_{-}^{(r)}\right|,\left|u_{+}^{(r)}-v_{+}^{(r)}\right|\right\},
$$


where $[v]^{r}=\left[v_{-}^{(r)}, v_{+}^{(r)}\right] ; u, v \in \mathbb{R}_{\mathcal{F}}$. We have that $D$ is a metric on $\mathbb{R}_{\mathcal{F}}$. Then $\left(\mathbb{R}_{\mathcal{F}}, D\right)$ is a complete metric space, see [7], with the properties

$$
\begin{aligned}
D(u \oplus w, v \oplus w) & =D(u, v), \quad \forall u, v, w \in \mathbb{R}_{\mathcal{F}}, \\
D(k \odot u, k \odot v) & =|k| D(u, v), \quad \forall u, v \in \mathbb{R}_{\mathcal{F}}, \forall k \in \mathbb{R}, \\
D(u \oplus v, w \oplus e) & \leq D(u, w)+D(v, e), \quad \forall u, v, w, e \in \mathbb{R}_{\mathcal{F}} .
\end{aligned}
$$

Let $f, g:[a, b] \rightarrow \mathbb{R}_{\mathcal{F}},[a, b] \subseteq \mathbb{R}$, be fuzzy real number valued functions. The distance between $f, g$ is defined by

$$
D^{*}(f, g):=\sup _{x \in[a, b]} D(f(x), g(x)) .
$$

Here $\sum^{*}$ stands for the fuzzy summation.

We use the following

Definition 2. Let $f:[a, b] \rightarrow \mathbb{R}_{\mathcal{F}}$ be a fuzzy real number valued function. We define the (first) fuzzy modulus of continuity of $f$ by

$$
\omega_{1}^{(\mathcal{F})}(f, \delta):=\sup _{\substack{x, y \in[a, b] \\|x-y| \leq \delta}} D(f(x), f(y)),
$$

any $0<\delta \leq b-a$.

Definition 3. Let $f:[a, b] \subseteq \mathbb{R} \rightarrow \mathbb{R}_{\mathcal{F}}$. We say that $f$ is fuzzy continuous at $x_{0} \in[a, b]$ iff whenever $x_{n} \rightarrow x_{0}$, then $D\left(f\left(x_{n}\right), f\left(x_{0}\right)\right) \rightarrow 0$, as $n \rightarrow \infty, n \in \mathbb{N}$. We call $f$ fuzzy continuous iff it is fuzzy continuous $\forall x \in[a, b]$ and we denote the space of fuzzy continuous functions by $C_{\mathcal{F}}([a, b])$.

The space $C_{\mathcal{F}}([a, b])$ is only a cone and not a vector space, however any finite linear combination of its elements with scalars in $\mathbb{R}$ belongs there.

Denote $[f]^{r}=\left[f_{-}^{(r)}, f_{+}^{(r)}\right]$ and we mean

$$
[f(x)]^{r}=\left[f_{-}^{(r)}(x), f_{+}^{(r)}(x)\right], \quad \forall x \in[a, b], \quad \text { all } r \in[0,1] .
$$

Let $f, g \in C_{\mathcal{F}}([a, b])$ we say that $f$ is fuzzy larger than $g$ pointwise and we denote it by $f \succsim g$ iff $f(x) \succsim g(x)$ iff $f_{-}^{(r)}(x) \geq g_{-}^{(r)}(x)$ and $f_{+}^{(r)}(x) \geq g_{+}^{(r)}(x), \forall x \in[a, b]$, $\forall r \in[0,1]$, iff $f_{-}^{(r)} \geq g_{-}^{(r)}, f_{+}^{(r)} \geq g_{+}^{(r)}, \forall r \in[0,1]$. 
Let $L$ be a map from $C_{\mathcal{F}}([a, b])$ into itself, we call it a fuzzy linear operator iff

$$
L\left(c_{1} \odot f_{1} \oplus c_{2} \odot f_{2}\right)=c_{1} \odot L\left(f_{1}\right) \oplus c_{2} \odot L\left(f_{2}\right),
$$

for any $c_{1}, c_{2} \in \mathbb{R}, f_{1}, f_{2} \in C_{\mathcal{F}}([a, b])$. We say that $L$ is a fuzzy positive linear operator iff for $f, g \in C_{\mathcal{F}}([a, b])$ with $f \succsim g$ we get $L(f) \succsim L(g)$ iff $(L(f))_{-}^{(r)} \geq(L(g))_{-}^{(r)}$ and $(L(f))_{+}^{(r)} \geq(L(g))_{+}^{(r)}$ on $[a, b]$ for all $r \in[0,1]$.

Example 1. Let $f \in C_{\mathcal{F}}([0,1])$, we define the fuzzy Bernstein operator

$$
\left(B_{n}^{(\mathcal{F})}(f)\right)(x)=\sum_{k=0}^{n} *\left(\begin{array}{l}
n \\
k
\end{array}\right) x^{k}(1-x)^{n-k} \odot f\left(\frac{k}{n}\right), \quad \forall x \in[0,1], n \in \mathbb{N} .
$$

This is a fuzzy positive linear operator.

We mention the very interesting with rates approximation motivating this work.

Theorem 1 (see p. 642, [2], S. Gal). If $f \in C_{\mathcal{F}}([0,1])$, then

$$
D^{*}\left(B_{n}^{(\mathcal{F})}(f), f\right) \leq \frac{3}{2} \omega_{1}^{(\mathcal{F})}\left(f, \frac{1}{\sqrt{n}}\right), \quad \forall n \in \mathbb{N}
$$

i.e.

$$
\lim _{n \rightarrow \infty} D^{*}\left(B_{n}^{(\mathcal{F})}(f), f\right)=0
$$

that is $B_{n}^{(\mathcal{F})} f \rightarrow f, n \rightarrow \infty$ in fuzzy uniform convergence.

The last fact comes by the property that $\omega_{1}^{(\mathcal{F})}(f, \delta) \rightarrow 0$ as $\delta \rightarrow 0$, whenever $f \in C_{\mathcal{F}}([a, b])$.

We need to use

Theorem 2 (Shisha and Mond (1968), [6]). Let $[a, b] \subseteq \mathbb{R}$. Let $\left(\tilde{L}_{n}\right)_{n \in \mathbb{N}}$ be a sequence of positive linear operators from $C([a, b])$ into itself. For $n=1,2, \ldots$, suppose $\tilde{L}_{n}(1)$ is bounded. Let $f \in C([a, b])$. Then for $n=1,2, \ldots$, we have

$$
\left\|\tilde{L}_{n} f-f\right\|_{\infty} \leq\|f\|_{\infty}\left\|\tilde{L}_{n} 1-1\right\|_{\infty}+\left\|\tilde{L}_{n}(1)+1\right\|_{\infty} \omega_{1}\left(f, \mu_{n}\right),
$$

where $\omega_{1}$ is the standard real modulus of continuity and

$$
\mu_{n}:=\left\|\left(\tilde{L}_{n}\left((t-x)^{2}\right)\right)(x)\right\|_{\infty}^{1 / 2},
$$


and $\|\cdot\|_{\infty}$ stands for the sup-norm over $[a, b]$. In particular, if $L_{n}(1)=1$ then

$$
\left\|\tilde{L}_{n} f-f\right\|_{\infty} \leq 2 \omega_{1}\left(f, \mu_{n}\right) .
$$

Note. One can easily see ([6]), for $n=1,2, \ldots$,

$$
\mu_{n}^{2} \leq\left\|\left(\tilde{L}_{n}\left(t^{2}\right)\right)(x)-x^{2}\right\|_{\infty}+2 c\left\|\left(\tilde{L}_{n}(t)\right)(x)-x\right\|_{\infty}+c^{2}\left\|\left(\tilde{L}_{n}(1)\right)(x)-1\right\|_{\infty},
$$

where $c:=\max (|a|,|b|)$.

Assuming that $\tilde{L}_{n}(1) \stackrel{u}{\longrightarrow} 1, \tilde{L}_{n}(i d) \stackrel{u}{\longrightarrow} i d, \tilde{L}_{n}\left(i d^{2}\right) \stackrel{u}{\longrightarrow} i d^{2}$ (id is the identity map), $n \rightarrow \infty$, uniformly, then from Theorem 2's main inequality we get $\tilde{L}_{n}(f) \stackrel{u}{\longrightarrow} f$, $\forall f \in C([a, b])$, that is the famous Korovkin theorem (see [5]) in the real case.

We finally need

Lemma 1. Let $f \in C_{\mathcal{F}}([a, b]),[a, b] \subseteq \mathbb{R}$. Then it holds

$$
\omega_{1}^{(\mathcal{F})}(f, \delta)=\sup _{r \in[0,1]} \max \left\{\omega_{1}\left(f_{-}^{(r)}, \delta\right), \omega_{1}\left(f_{+}^{(r)}, \delta\right)\right\},
$$

for any $0<\delta \leq b-a$.

Proof. Let $x, y \in[a, b]:|x-y| \leq \delta, 0<\delta \leq b-a$. Then we have

$$
\begin{aligned}
D(f(x), f(y)) & =\sup _{r \in[0,1]} \max \left\{\left|(f(x))_{-}^{(r)}-(f(y))_{-}^{(r)}\right|,\left|(f(x))_{+}^{(r)}-(f(y))_{+}^{(r)}\right|\right\} \\
& \leq \sup _{r \in[0,1]} \max \left\{\omega_{1}\left(f_{-}^{(r)}, \delta\right), \omega_{1}\left(f_{+}^{(r)}, \delta\right)\right\} .
\end{aligned}
$$

Thus

$$
\omega_{1}^{(\mathcal{F})}(f, \delta) \leq \sup _{r \in[0,1]} \max \left\{\omega_{1}\left(f_{-}^{(r)}, \delta\right), \omega_{1}\left(f_{+}^{(r)}, \delta\right)\right\} .
$$

For any $r \in[0,1]$ and any $x, y \in[a, b]:|x-y| \leq \delta$ we see that

$$
\omega_{1}^{(\mathcal{F})}(f, \delta) \geq D(f(x), f(y)) \geq\left|(f(x))_{-}^{(r)}-(f(y))_{-}^{(r)}\right|,\left|(f(x))_{+}^{(r)}-(f(y))_{+}^{(r)}\right| .
$$

Therefore

$$
\omega_{1}\left(f_{ \pm}^{(r)}, \delta\right) \leq \omega_{1}^{(\mathcal{F})}(f, \delta), \quad \forall r \in[0,1]
$$

Hence

$$
\sup _{r \in[0,1]} \max \left\{\omega_{1}\left(f_{-}^{(r)}, \delta\right), \omega_{1}\left(f_{+}^{(r)}, \delta\right)\right\} \leq \omega_{1}^{(\mathcal{F})}(f, \delta),
$$

proving the claim. 
Note. For $f \in C_{\mathcal{F}}([a, b])$ we get that $f$ is fuzzy bounded and $\omega_{1}^{(\mathcal{F})}(f, \delta)$ is finite for all $0<\delta \leq b-a$. Also $f_{ \pm}^{(r)}$ are continuous on $[a, b]$ and $\omega_{1}\left(f_{ \pm}^{(r)}, \delta\right)$ are finite too, all $r \in[0,1]$.

\section{Main Results}

We present the fuzzy analog of Shisha-Mond inequality of Theorem 2.

Theorem 3. Let $\left\{L_{n}\right\}_{n \in \mathbb{N}}$ be a sequence of fuzzy positive linear operators from $C_{\mathcal{F}}([a, b])$ into itself, $[a, b] \subseteq \mathbb{R}$. We assume that there exists a corresponding sequence $\left\{\tilde{L}_{n}\right\}_{n \in \mathbb{N}}$ of positive linear operators from $C([a, b])$ into itself with the property

$$
\left(L_{n}(f)\right)_{ \pm}^{(r)}=\tilde{L}_{n}\left(f_{ \pm}^{(r)}\right)
$$

respectively, $\forall r \in[0,1], \forall f \in C_{\mathcal{F}}([a, b])$. We assume that $\left\{\tilde{L}_{n}(1)\right\}_{n \in \mathbb{N}}$ is bounded. Then for $n \in \mathbb{N}$ we have

$$
D^{*}\left(L_{n} f, f\right) \leq\left\|\tilde{L}_{n} 1-1\right\|_{\infty} D^{*}(f, \tilde{o})+\left\|\tilde{L}_{n}(1)+1\right\|_{\infty} \omega_{1}^{(\mathcal{F})}\left(f, \mu_{n}\right),
$$

where

$$
\mu_{n}:=\left(\left\|\left(\tilde{L}_{n}\left((t-x)^{2}\right)\right)(x)\right\|_{\infty}\right)^{1 / 2},
$$

$\forall f \in C_{\mathcal{F}}([a, b]), \tilde{o}:=\mathcal{X}_{\{0\}}$ the neutral element for $\oplus$. If $\tilde{L}_{n} 1=1, n \in \mathbb{N}$, then

$$
D^{*}\left(L_{n} f, f\right) \leq 2 \omega_{1}^{(\mathcal{F})}\left(f, \mu_{n}\right)
$$

Note. The fuzzy Bernstein operators $B_{n}^{(\mathcal{F})}$ and the real corresponding ones $B_{n}$ acting on $C_{\mathcal{F}}([0,1])$ and $C([0,1])$, respectively, fulfill assumption (1).

We present now the Fuzzy Korovkin Theorem.

Theorem 4. Let $\left\{L_{n}\right\}_{n \in \mathbb{N}}$ be a sequence of fuzzy positive linear operators from $C_{\mathcal{F}}([a, b])$ into itself, $[a, b] \subseteq \mathbb{R}$. We assume that there exists a corresponding sequence $\left\{\tilde{L}_{n}\right\}_{n \in \mathbb{N}}$ of positive linear operators from $C([a, b])$ into itself with the property

$$
\left(L_{n}(f)\right)_{ \pm}^{(r)}=\tilde{L}_{n}\left(f_{ \pm}^{(r)}\right),
$$


respectively, $\forall r \in[0,1], \forall f \in C_{\mathcal{F}}([a, b])$. Furthermore assume that

$$
\tilde{L}_{n}(1) \stackrel{u}{\longrightarrow} 1, \quad \tilde{L}_{n}(i d) \stackrel{u}{\longrightarrow} i d, \quad \tilde{L}_{n}\left(i d^{2}\right) \stackrel{u}{\longrightarrow} i d^{2},
$$

as $n \rightarrow \infty$, uniformly. Then

$$
D^{*}\left(L_{n} f, f\right) \longrightarrow 0, \quad \text { as } n \rightarrow \infty,
$$

for any $f \in C_{\mathcal{F}}([a, b])$, i.e. $L_{n} f \stackrel{D^{*}}{\longrightarrow} f$, that is $L_{n} \rightarrow I$ unit operator in the fuzzy sense, as $n \rightarrow \infty$.

Proof. Use of (2), property of (3), etc.

Example for Theorem 4 the fuzzy Bernstein operators $B_{n}^{(\mathcal{F})}$.

Proof of Theorem 3. We would like to estimate

$$
\begin{aligned}
D^{*}\left(L_{n} f, f\right)= & \sup _{x \in[a, b]} D\left(\left(L_{n} f\right)(x), f(x)\right) \\
= & \left.\sup _{x \in[a, b]} \sup _{r \in[0,1]} \max \left\{\left|\left(L_{n} f\right)_{-}^{(r)}(x)-(f)_{-}^{(r)}(x)\right|, \mid\left(L_{n} f\right)\right)_{+}^{(r)}(x)-(f)_{+}^{(r)}(x) \mid\right\} \\
= & \sup _{x \in[a, b]} \sup _{r \in[0,1]} \max \left\{\mid \tilde{L}_{n}\left(f_{-}^{(r)}(x)-(f)_{-}^{(r)}(x)|,| \tilde{L}_{n}\left(f_{+}^{(r)}\right)(x)-(f)_{+}^{(r)}(x) \mid\right\}\right. \\
= & \sup _{r \in[0,1]} \max \left\{\left\|\tilde{L}_{n} f_{-}^{(r)}-f_{-}^{(r)}\right\|_{\infty},\left\|\tilde{L}_{n} f_{+}^{(r)}-f_{+}^{(r)}\right\|_{\infty}\right\} \\
\leq & \sup _{r \in[0,1]} \max \left\{\left(\left\|f_{-}^{(r)}\right\|_{\infty}\left\|\tilde{L}_{n} 1-1\right\|_{\infty}+\left\|\tilde{L}_{n}(1)+1\right\|_{\infty} \omega_{1}\left(f_{-}^{(r)}, \mu_{n}\right)\right),\right. \\
\leq & \left\|\tilde{L}_{n} 1-1\right\|_{\infty} \sup _{r \in[0,1]} \max \left(\left\|f_{-}^{(r)}\right\|_{\infty},\left\|f_{+}^{(r)}\right\|_{\infty}\right) \\
& +\left\|\tilde{L}_{n}(1)+1\right\|_{\infty} \sup _{r \in[0,1]} \max \left\{\omega_{1}\left(f_{-}^{(r)}, \mu_{n}\right), \omega_{1}\left(f_{+}^{(r)}, \mu_{n}\right)\right\} \\
\quad(\operatorname{by} \operatorname{Lemma} 1) & \left\|\tilde{L}_{n} 1-1\right\|_{\infty} D^{*}(f, \tilde{o})+\left\|\tilde{L}_{n}(1)+1\right\|_{\infty} \omega_{1}^{(\mathcal{F})}\left(f, \mu_{n}\right),
\end{aligned}
$$

proving (2). 
Application 1. Let $f \in C_{\mathcal{F}}([0,1])$ then by applying (2) we obtain

$$
D^{*}\left(B_{n}^{(\mathcal{F})} f, f\right) \leq 2 \omega_{1}^{(\mathcal{F})}\left(f, \frac{1}{2 \sqrt{n}}\right), \quad \forall n \in \mathbb{N} .
$$

\section{References}

[1] Anastassiou, G.A., Moments in Probability and Approximation Theory, Pitman/ Longman, \#287, UK, 1993.

[2] Gal, S., Approximation theory in Fuzzy setting, Chapter 13, pp. 617-666, in Handbook of Analytic-Computational Methods in Applied Mathematics, editor, G.A. Anastassiou, Chapman \& Hall/CRC, Boca Raton, 2000.

[3] Goetschel Jr., R., and Voxman, W., Elementary fuzzy calculus, Fuzzy Sets and Systems, 18 (1986), 31-43.

[4] Kaleva, O., Fuzzy differential equations, Fuzzy Sets and Systems, 24 (1987), 301-317.

[5] Korovkin, P.P., Linear Operators and Approximation Theory, Hindustan Publ. Corp., Delhi, India, 1960.

[6] Shisha, O., and Mond, B., The degree of convergence of sequences of linear positive operators, Nat. Acad. of Sci. U.S., 60 (1968), 1196-1200.

[7] Congxin $\mathrm{Wu}$, and Ming Ma, On embedding problem of fuzzy number space: Part 1 , Fuzzy Sets and Systems, 44 (1991), 33-38.

[8] Congxin Wu and Zengtai Gong, On Henstock integral of fuzzy-number-valued functions (I), Fuzzy Sets and Systems, 120 (2001), 523-532.

Department of Mathematical Sciences, The University of Memphis, Memphis, TN 38152, U.S.A.,

E-mail address: ganastss@memphis.edu 